\title{
The nutrition support needs of cancer survivors in Ireland
}

\author{
C.M. Timon ${ }^{1}$ and S. Doyle ${ }^{2}$ \\ ${ }^{1}$ School of Nursing, Psychotherapy and Community Health, Dublin City University, Dublin 9, Ireland and \\ ${ }^{2}$ School of Biological \& Health Sciences, Technological University Dublin, Dublin 7, Ireland
}

Cancer patients and survivors face several challenges relating to diet including misinformation about diet and cancer and a reported

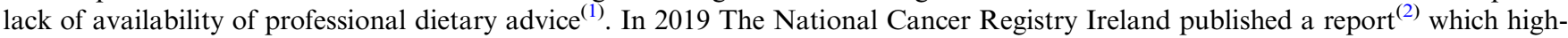
lighted the lack of support services for an array of physical and psychological issues affecting cancer survivors, including a lack of nutrition support. This research aims to investigate the type of nutrition support received, current dietary habits and preferred nutrition support going forward amongst cancer survivors in Ireland.

An online survey exploring the nutrition support needs of cancer survivors in Ireland was developed using LimeSurvey. Ethical approval was obtained from the DCU Research Ethics Committee (DCUREC/2020/012). Data collection commenced in March 2020 and is ongoing. Eligibility criteria included that respondents were: $\geq 18 y$ rs, living in Ireland, and $\geq 1$ year post primary cancer treatment. To date, 76 respondents have completed the survey. Responses were exported to SPSS V25 and descriptive statistics were applied to examine common attitudes amongst respondents.

The majority of the study population were female $(\mathrm{n}=68,89 \%)$, received third level education $(\mathrm{n}=48,63 \%)$ and aged between 45 and 64 years $(n=44,58 \%)$. Seventy-four percent $(n=56)$ of respondents have not received any nutrition support since their cancer treatment ceased and 65\% $(n=49)$ of respondents are still facing dietary issues as a result of their cancer and treatment $($ loss of appetite, loss of enjoyment of food). Twenty respondents reported that they received dietary support as a cancer survivor. The most common sources of dietary support included: a doctor/nurse $(n=9,45 \%)$, a dietitian $(n=8,40 \%)$ and a nutritional therapist $(n=4,20 \%)$, some respondents received dietary support from more than one source. All respondents that received dietary support reported they were advised to follow a healthy balanced diet $(n=20,100 \%)$ but in some cases were advised to avoid certain foods (advised to avoid: sweets/chocolate $(n=13,65 \%$ of respondents) processed meat $(n=11,55 \%$ of respondents), dairy ( $n=5,25 \%$ of respondents) and red meat $(n=4,20 \%$ of respondents)). Twenty-three percent $(n=17)$ of respondents felt their current diet is worse now than before their cancer diagnosis and wished for dietary support relating to practical information on how to achieve recommended nutrition guidelines $(n=55,73 \%$ of respondents), examples of recipes for healthy living $(n=50,66 \%$ of respondents) and clarification on misinformation relating to diet and cancer $(n=40,53 \%$ of respondents). With regards to the provision of nutrition support, $66 \%$ ( $n=$ $50)$ of respondents found information in the format of books/leaflets useful, however $88 \%(n=67)$ preferred the idea of an interactive online tool for the provision of support.

This research highlights the challenges facing cancer survivors in Ireland and provides useful insight into how nutrition support services could help address their needs.

1. Grunfeld E, Julian JA, Pond G, et al. (2011) J Clin Oncol 29, 36, 4755-62.

2. O'Connor M, O'Donovan B, Drummond F, Donnelly C. (2019) National Cancer Registry Ireland. 